\title{
Respiratory failure due to opportunistic diseases in AIDS
}

\author{
Insuficiência respiratória associada a doenças oportunísticas em AID S
}

Antônio Lúcio Teixeira Júnior ${ }^{1}$, Vandack Nobre ${ }^{2}$ and José Roberto Lambertucci ${ }^{2}$
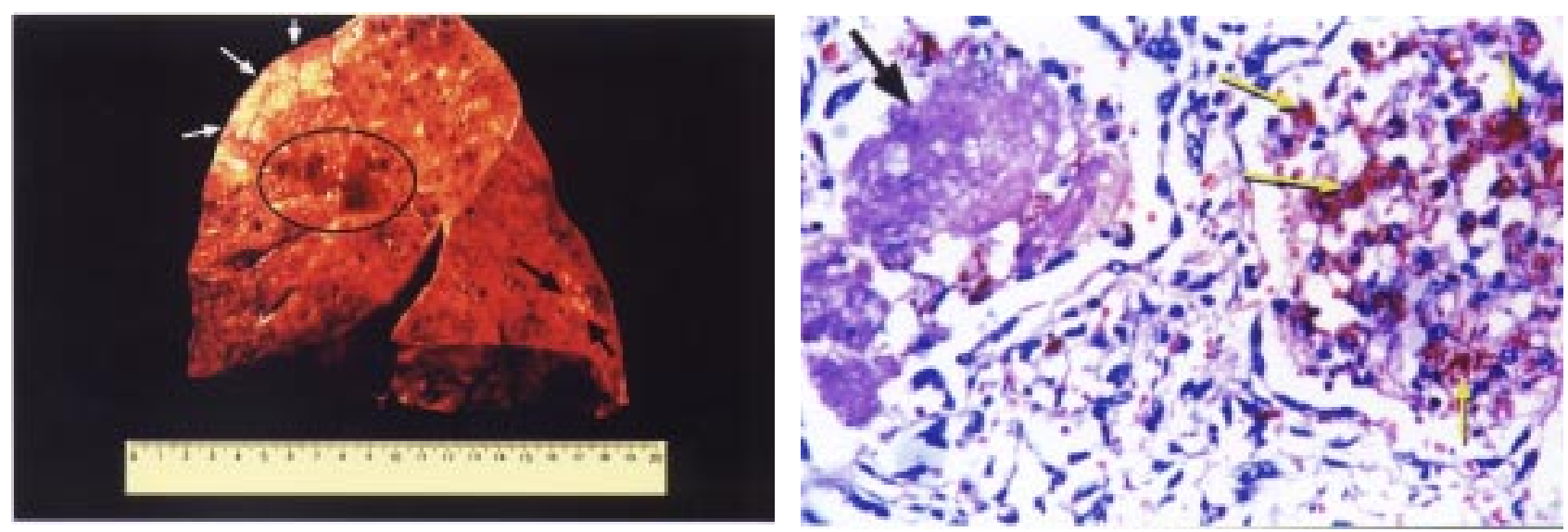

A

B

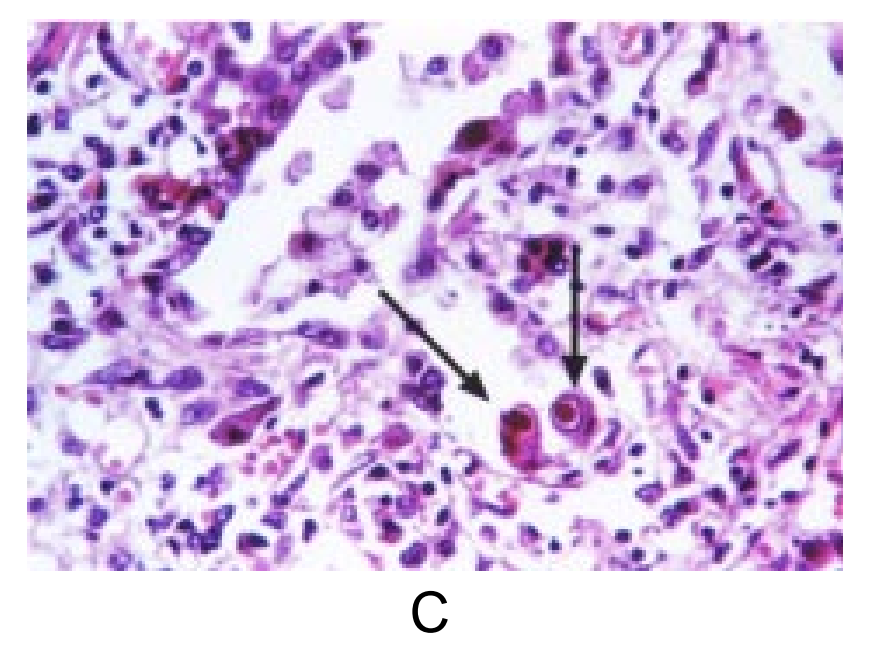

1. Departamento de Neurologia e Psiquiatria da Faculdade de Medicina da Universidade Federal de Minas Gerais, Belo Horizonte, MG. 2. Departamento de Clínica Médica da Faculdade de Medicina da Universidade Federal de Minas Gerais, Belo Horizonte, MG.

Endereço para correspondência: Dr. José Roberto Lambertucci. Deptº de Clínica Médica/FM/UFMG. Av. Alfredo Balena 190, 30130-100, Belo Horizonte, MG.

Recebido para publicação em 8/4/2002. 
A 31-year-old man was admitted to the emergency room of a university hospital of Belo Horizonte, MG, with a history of fever, dyspnea, and cough with purulent sputum starting three weeks before. He also reported a $10 \mathrm{~kg}$ weight loss since the onset of his symptoms and, in addition, two recent episodes of community-acquired pneumonia. Physical examination revealed disseminated red-purple plaques all over his body, generalized lymphadenopathy and signs which suggested the diagnosis of systemic inflammatory response syndrome (SIRS). Chest x-ray revealed diffuse opacity of both lung fields. Respiratory failure and hemodynamic instability required, respectively, mechanical ventilation and vasopressor therapy. Despite clinical support, administration of antibiotics and endovenous corticosteroids, the patient showed progressive clinical deterioration, dying six days after admission. HIV tests resulted positive. Autopsy revealed severe Pneumocystis carinii pneumonia (Figure A - white arrows and Figure B - black arrow), Kaposi's sarcoma (Figure A - brownish area inside of a circle) and Mycobacterium sp. The latter was found in abscesses situated in the inferior lobe of the right lung (Figure A black arrows and Figure B - yellow arrows; Wade X400) and were also retrieved from a satellite hilar lymph node. Cells with enlarged nucleoli and intranuclear inclusions ("bird eyes"), suggesting cytomegalovirus infection, were extensively distributed in both lungs (Figure $\mathrm{C}$ - arrows; HE X400). Staphylococcus aureus and Pseudomonas aeruginosa were cultured in the material obtained from multiple foci of nonspecific pneumonia. Microscopic examination of the lungs were compatible with the diagnosis of acute respiratory distress syndrome (ARDS).
O paciente, de 31 anos de idade, foi admitido ao pronto atendimento de um hospital universitário de Belo Horizonte, MG, com história de febre, dispnéia e tosse produtiva, iniciados há três semanas. Ele informava emagrecimento de $10 \mathrm{~kg}$ desde o início dos sintomas e, ainda, dois episódios recentes de pneumonia comunitária. Ao exame clínico evidenciou-se placas violáceas disseminadas pelo corpo, linfoadenomegalia generalizada e sinais sugestivos de síndrome da resposta inflamatória sistêmica (SIRS). A radiografia do tórax mostrou opacificação difusa dos campos pulmonares. Houve rápida progressão para insuficiência respiratória e choque, sendo instituídos ventilação mecânica, aminas vasoativas, antimicrobianos e corticoesteróide endovenoso. A despeito dessas medidas, houve piora clínica progressiva, culminando com a morte do paciente, seis dias após a admissão hospitalar. A sorologia para o HIV revelou-se positiva. No estudo anatomopatológico dos pulmões, destacaram-se extenso acometimento pelo sarcoma de Kaposi (Figura A - área amarronzada dentro do círculo) e pneumocistose (Figura A - setas brancas e Figura B seta preta; Wade X400). Isolou-se o Mycobacterium sp em abscessos do lobo inferior do pulmão direito (Figura A - setas negras e Figura B - setas amarelas; Wade $\mathrm{X} 400$ ) e em linfonodo hilar satélite. Células com nucléolos proeminentes e inclusões nucleares ("olho de ave"), sugestivas de citomegalovirose, encontravam-se difusamente distribuídas nos pulmões (Figura $\mathrm{C}$ - setas; HE X400). Havia, ainda, pneumonia inespecífica multifocal, de onde isolaram-se Staphylococcus aureus e Pseudomonas aeruginosa. Ao exame microscópico dos pulmões notou-se alterações sugestivas de síndrome da angústia respiratória do adulto (SARA).

\section{REFERÊNCIAS BIBLIOGRÁFICAS}

1. Brito AM, Castilho EA, Szwarcwald CL. AIDS e infecção pelo HIV no Brasil: uma epidemia multifacetada. Revista da Sociedade Brasileira de Medicina Tropical 34: 207-217, 2000.

2. Jasmer RM, Edinburgh KJ, Thompson A, Gotway MB, Creasman JM, Webb WR, Huang L. Clinical and radiographic predictors of the etiology of pulmonary nodules in HIV-infected patients. Chest 117:1023-1029, 2000
3. Lambertucci JR, Rayes AA, Nunes F, Landazuri JP, Nobre V. Fever of undetermined origin in patients with acquired immunodeficiency syndrome. Revista do Instituto de Medicina Tropical de São Paulo 41: 27-32, 1999. 